\title{
Position Control of Motor Drive Systems: A Data Driven Approach
}

\author{
Hossein Parastvand ${ }^{1}$, Mohammad Javad Khosrowjerdi ${ }^{1}$, Nacer Kouider M'Sirdi ${ }^{2}$ \\ ${ }^{1}$ Advanced Control Research Laboratory (ACRL), Department of Electrical Engineering Sahand University of \\ Technology (SUT) Tabriz, Iran \\ ${ }^{2}$ Aix Marseille Université, CNRS, ENSAM, Université de Toulon, LSIS UMR 7296 LSIS UMR CNRS UMR, Domaine \\ Universitaire St Jérôme, Avenue Escadrille Normandie-Niemen, 13397 Marseille Cedex 20, France \\ h_parastvand@sut.ac.ir, khosrowjerdi@sut.ac.ir, Nacer.msirdi@lsis.org
}

\begin{abstract}
A new model-free approach for designing robust PID controllers for the position control of electrical machines (such as induction, synchronous or DC motors) with un-modeled dynamics is proposed.

In this paper, it is illustrated that frequency response data is sufficient to calculate a family of robust PID controllers that satisfy an $\boldsymbol{H}_{\infty}{ }^{-}$norm on the complementary sensitivity function. The approach is illustrated on an induction motor drive system through simulation.
\end{abstract}

Keywords- motor drive, position control, PID controller, robust control, frequency domain, model free approach;

\section{INTRODUCTION}

Motion control applications can be found in almost every part of industry by induction motor, synchronous motor and DC motor. They are used to regulate mechanical motions in terms of position, velocity and acceleration. A high performance motor drive must have good position command, tracking and load to regulate response in the presence of external load uncertainty, un-modeled and nonlinear dynamics of plant. Similar to other industrial control problems, PID is the first choice in most of motion control applications.

In [1] the PID controller has been designed based on stability analysis considering passivity in null space motion of redundant manipulator. A selftuning PID control strategy has been proposed in [2] for implementing a motion control system that stabilizes the two-wheeled vehicle and follows the desired motion commands. The controller parameters are tuned automatically, on-line, to overcome the disturbances and parameter variations. In [3] the dynamics of the synchronous motor has been investigated and the motor's response to rapid load changes has been analyzed using decentralized PID controller. Another modelbased application with PID has been proposed in [4] on load motion control of two-mass servomechanisms. Although most of the proposed methods in this area rely on plant mathematical model, yet some model-free approaches can be found in the literature such as $[5,6,7,8,18,19]$. Almost every proposed model-free approach uses time domain data and online adaptive algorithms. A PID position domain control (PDC) is proposed in [6] for reducing contour tracking errors. Fuzzy control is another model-free approach to motion control of motor drives $[9,10]$. An intelligent PID controller has been proposed in $[18,19]$ based on a newly developed numerical differentiation. The approach use time domain and numerical differentiation to adjust the control.

In this paper, another model-free technique is proposed in frequency domain for robust PID controller design of motor drive systems faced to un-modeled dynamics that can be classified as structured uncertainties. The algorithm used to calculate the family of stabilizing PID controllers for nominal plant proposed in [11] is extended to plants with an uncertain parameter. It is shown that the only required data is the set of frequency responses of motor drive system including frequency responses corresponding to maximum and minimum values of uncertain parameter. Also, the family of robust PID controllers that achieve $H_{\infty}$ norm on complementary sensitivity function is calculated. Some special applications of the proposed approach are reviewed. It is shown that the problem can be solved with more relax conditions if the uncertain parameter appears in the feedforward path.

The paper is organized as follows. The position control problem is introduced in Section II. The algorithm used to calculate the family of stabilizing PID controllers for nominal plants and its extension to uncertain plants are presented in Section III. A review on special cases of application of the proposed approach is presented in Section IV. The proposed approach is simulated on induction motor drive in Section V. Finally, some concluding remarks are given in Section VI.

\section{PROBLEM FORMULATION}

The basic model for many industrial motor drives can be described by an electrical part and a mechanical part [10]. This structure is shown in Fig. 1. The electrical part is placed between input voltage $u$ and output torque $T_{e}$ and the mechanical part is between torque $T_{e}$ and the rotor position $\theta$. The electrical part that is much faster than the other one can be approximated by a first order transfer function plus a small time delay; and the mechanical part of electrical motors can be described by a twoorder transfer function [10]. In Fig. 1 the transfer 
function " $W(s) \Delta$ " where $\Delta$ is an unknown constant and $|\Delta|<1$ represents for the un-modeled dynamics.

The paper investigates the position control of electrical motor drives that can be configured as structure of Fig. 1. This problem is formulated as follows. Consider the basic structure of a motor drive system and assume that

1. The frequency response from voltage $u$ to rotor position $\theta$ is available.

2. $\Delta$ is an unknown constant and $|\Delta|<1$.

Under these assumptions the control objective is to calculate the family of PID controllers that stabilize the uncertain plants $P(s)(1+W(s) \Delta)$ where $|\Delta|<1$ and satisfies $H_{\infty}$-norm consideration on complementary sensitivity function in the presence of un-modeled dynamics.

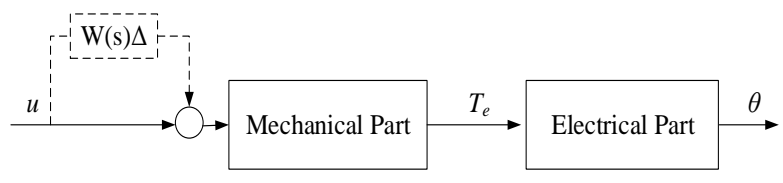

Fig. 1. Motor drive system with un-modeled dynamics

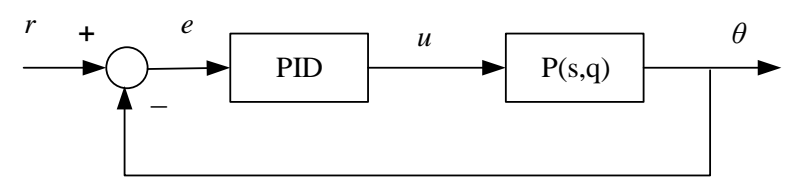

Fig. 2. Feedback structure with uncertain plant and PID

Fig. 2 shows the feedback system with PID controller in which $P(s, q)$ is an uncertain plant and $q$ can be substituted by $\Delta$. In the next section, a survey on the algorithm proposed for nominal stability in [11] and its generalization to uncertain plants is presented and it is illustrated that the control objectives of this paper on motor drive system can be satisfied in a same way.

\section{PID CONTROLLER DESIGN: MODEL FREE APPROCH}

In this section, the problem of achieving the family of stabilizing PID controllers for nominal stable plant $P(s)$ proposed in [11] is reviewed. Then by verifying a theorem, this approach is generalized to plants with an uncertain parameter. Similar approach is proposed for unstable plants which are omitted here because of room limitation.

The feedback system with PID controller is shown in Fig. 2. First, some mathematical preliminaries are introduced. Consider a real rational function

$$
P(s)=\frac{A(s)}{B(s)}
$$

where $A(s)$ and $B(s)$ are polynomials with real coefficients and of degrees $m$ and $n$, respectively. Assume that $A(s)$ and $B(s)$ have no zero on $j \omega$ axis. Let $z^{+}, p^{+}\left(z^{-}, p^{-}\right)$determine the number of open right halp plane (RHP) (open left half plane (LHP)) zeros and poles of $P(s)$. Also let $\left.\Delta\right|_{0} ^{\infty}(P(j \omega))$ denotes the net change in phase of $P(s)$ as $\omega$ runs from 0 to $+\infty$. Then

$$
\left.\Delta\right|_{0} ^{\infty}(P)=\frac{\pi}{2}\left[z^{-}-z^{+}-\left(p^{-}-p^{+}\right)\right]
$$

Define the (Hurwitz) signature of $P(s)$ as

$$
\sigma(P)=\left.\frac{2}{\pi} \Delta\right|_{0} ^{\infty}(P(j \omega))
$$

and since $P(s)$ has no pole and zero on $j \omega$ axis, it can be written

$$
\sigma(P)=-(n-m)-2\left(z^{+}-p^{+}\right)
$$

The value of $n-m$ can be calculated from the frequency data of $P(s)$ and $z^{+}$can be calculated from (3). Let

$$
P(j \omega)=P_{r}(j \omega)+j P_{i}(j \omega)
$$

where $P_{r}(j \omega)$ and $P_{i}(j \omega)$ denote the real and imaginary parts of $P(j \omega)$, respectively. Assume that the real, distinct, finite zeros of $P_{i}(j \omega)=0$ denote as $\omega_{0}, \omega_{1}, \ldots, \omega_{l-1}$ such that

$$
0=\omega_{0}<\omega_{1}<\cdots<\omega_{l-1}
$$

and consider the modified PID controller as

$$
C(s)=\frac{k_{i}+k_{p} s+k_{d} s^{2}}{s(1+s T)}
$$

where $k_{p}, k_{i}$ and $k_{d}$ are the proportional, integral and derivative coefficients of PID controller and $T$ is a positive constant.

Lemma 1: Let

and

$$
F(s):=s(1+s T)+\left(k_{i}+k_{p} s+k_{d} s^{2}\right) P(s)
$$

$\bar{F}(s)=F(s) P(-s)$.

Then the closed loop stability is equal to

$$
\sigma(\bar{F}(s))=n-m+2 z^{+}+2 .
$$

Let

where

$$
\bar{F}(j \omega)=\bar{F}_{r}\left(\omega, k_{i}, k_{d}\right)+j \omega \bar{F}_{i}\left(\omega, k_{p}\right)
$$

$$
\begin{aligned}
\bar{F}_{r}\left(\omega, k_{i}, k_{d}\right)= & \left(k_{i}-k_{d} \omega^{2}\right)|P(j \omega)|^{2}-\omega^{2} T P_{r}(\omega) \\
& +\omega P_{i}(\omega)
\end{aligned}
$$

and

$$
\bar{F}_{i}\left(\omega, k_{p}\right)=k_{p}|P(j \omega)|^{2}+P_{r}(\omega)+\omega T P_{i}(\omega)
$$

Consider $\bar{F}_{i}\left(\omega, k_{p}\right)=0$ and define

$$
k_{p}=-\frac{\cos \phi(\omega)+\omega T \sin \phi(\omega)}{|P(j \omega)|}:=g(\omega)
$$

and $J=\operatorname{sgn}\left[\bar{F}_{i}\left(\infty^{-}, k_{p}\right)\right]$ where $k_{p}^{\min }<k_{p}^{*}<k_{p}^{\max }$.

Theorem 1: Let $\omega_{1}<\omega_{2}<\cdots<\omega_{l-1}$ denote the distinct frequencies of odd multiplicities which are solutions of

$$
\bar{F}_{i}\left(\omega, k_{p}^{*}\right)=0 .
$$

Determine strings of integers $I=\left[i_{0}, i_{1}, i_{2}, \ldots, i_{l}\right]$ where $i_{t} \in\{-1,1\}$ such that: 
for $n-m$ even:

$$
\begin{gathered}
{\left[i_{0}-i_{1}+i_{2}+\cdots+(-1)^{l-1} 2 i_{l-1}+(-1)^{l} i_{l}\right](-1)^{l-1} J} \\
=n-m+2 z^{+}+2
\end{gathered}
$$

and for $n-m$ odd:

$\left[i_{0}-i_{1}+i_{2}+\cdots+(-1)^{l-1} 2 i_{l-1}\right](-1)^{l-1} J$

$$
=n-m+2 z^{+}+2
$$

Also let

$$
i_{l}=\operatorname{sgn}\left(\bar{F}_{r}\left(\infty^{-}, k_{i}, k_{d}\right) .\right.
$$

Then for $k_{p}=k_{p}^{*}$, the values of $\left(k_{i}, k_{d}\right)$ corresponding to the closed loop stability are given by

$$
\bar{F}_{r}\left(\omega_{t}, k_{i}, k_{d}\right) i_{t}>0
$$

where $i_{t}$ 's are taken from strings satisfy (5) or (6) and $\omega_{t}{ }^{\prime} s$ are taken from the solutions of (4).

Next theorem shows how to calculate the admissible range of $k_{p}$.

Theorem 2: The necessary condition of existence the stabilizing PID for LTI plants is that there exists $k_{p}$ such that $k_{p}=g(\omega)$ has at least $R$ distinct roots of odd multiplicities such that

$$
\left\{\begin{array}{ll}
R \geq \frac{n-m+2 z^{+}+2}{2}-1 & n-m \text { even } \\
R \geq \frac{n-m+2 z^{+}+3}{2}-1 & n-m \text { odd }
\end{array} .\right.
$$

The procedure for calculation the family of stabilizing PID controllers is summarized in the following algorithm [11].

\section{Algorithm 1.}

1. Determine the relative degree $r_{P}$ of plant $P(s)$ from high frequency slope of bode magnitude of $P(j \omega)$.

2. Determine $\sigma(P)$ from (2).

3. Determine $z^{+}$from (3).

4. Determine $g(\omega)$ for $\omega \geq 0$ from (4).

5. Apply Theorem 2 to determine the range of $k_{p}$.

6. For $k_{p}=k_{p}^{*}$, solve (4) and obtain $\omega_{1}<\omega_{2}<$ $\cdots<\omega_{l-1}$.

7. Let $\omega_{0}=0$ and $\quad \omega_{l}=\infty$. Determine $i_{0}, i_{1}, \ldots, i_{l-1}$ from (5) or (6).

8. For $k_{p}=k_{p}^{*}$, determine the $\left(k_{i}, k_{d}\right)$ values from (7).

9. Change $k_{p}$ and go to step 6 to obtain the whole family of stabilizing PID controllers.

Now the main result of this paper will be presented. Consider an uncertain real rational plant $P(s, q)$ where $q \in\left[q_{\min }, q_{\max }\right]$ is an uncertain parameter. The control goal is to calculate the family of robust stabilizing PID controllers for the uncertain plant $P(s, q)$. The feedback structure is shown in Fig. 2. The only required data is the set of frequency responses $P(j \omega, q)$ including $P\left(j \omega, q_{\min }\right)$ and $P\left(j \omega, q_{\max }\right)$ for $\omega \geq 0$.

In the proposed approach of Algorithm 1, roots $R$ of function $g(\omega)$ in (8), can be obtained from (4) and the range of $k_{p}$ is calculated based on the value of $R$. Also the range of admissible $\left(k_{i}, k_{d}\right)$ values obtain from (7) which have the slope equal to $\omega_{t}^{2}$. If by monotonic variation of the uncertain parameter $q$, $g(\omega)$ varies monotonically, some substantial results could be concluded. First of all, the range of admissible $k_{p}$ for stabilizing the uncertain plant $P(s, q)$ is the common range of admissible $k_{p}$ for two plants $P\left(s, q_{\min }\right)$ and $P\left(s, q_{\max }\right)$. Second, since the slope of linear inequalities in (7) varies monotonically with monotonic varying the uncertain parameter, the inequalities corresponding to $P\left(s, q_{\min }\right)$ and $P\left(s, q_{\max }\right)$ have the maximum and minimum slopes, not necessarily respectively. Finally, since variation of the uncertain parameter $q$, does not lead to monotonic variation in the $y-$ intercept, the common space of inequality (7) for $P\left(s, q_{\min }\right)$ and $P\left(s, q_{\max }\right)$ is larger than the admissible $\left(k_{i}, k_{d}\right)$ values. An approach to find the exact range of admissible parameters is presented in the following remark.

Remark 1: A simple approach to exclude these regions is to choose some test points in those regions and analyzing their stability. So monotonic variation of the function $g(\omega)$, makes it easy to calculate the family of stabilizing PID controllers for plants with an uncertain parameter.

Theorem 3: Let $P(s, q)$ be a real rational transfer function and $q \in\left[q_{\min }, q_{\max }\right]$ is an uncertain parameter. If the Number of zeros and poles and the number of RHP zeros of $P(s, q)$ are fixed for $q \in\left[q_{\min }, q_{\max }\right]$ then the family of stabilizing PID controllers for uncertain plant is a subspace of common space between two set of stabilizing PID controllers for $P\left(s, q_{\min }\right)$ and $P\left(s, q_{\max }\right)$ if one of the below constraints satisfy

$$
\begin{gathered}
\left\{\begin{array}{c}
p_{i}^{2}+P_{r}^{2}+P_{i} \omega+P_{r}<0 \\
-\left(P_{r}+P_{i} \omega\right)>0
\end{array}\right. \\
\left\{\begin{array}{c}
p_{i}^{2}+P_{r}^{2}+P_{i} \omega+P_{r}>0 \\
-\left(P_{r}+P_{i} \omega\right)<0
\end{array}\right.
\end{gathered}
$$

for only one value of uncertain parameter $q$ and for $\omega \geq 0$.

Proof: If monotonic varying the uncertain parameter leads to monotonic varying the function $g(\omega$ then the family of stabilizing PID controllers for uncertain plant is a subspace of common space between two set of stabilizing PID controllers for $P\left(s, q_{\min }\right)$ and $P\left(s, q_{\max }\right)$. Then 


$$
\frac{d g}{d k}=-\frac{\left(\frac{d P_{r}}{d k}+\omega \frac{d P_{i}}{d k}\right)\left(p_{i}^{2}+P_{r}^{2}\right)-2\left(P_{r} \frac{d P_{r}}{d k}+P_{i} \frac{d P_{i}}{d k}\right)\left(P_{r}+P_{i} \omega\right)}{p_{i}^{2}+P_{r}^{2}}
$$

Without loss of generality, consider the case of monotonic increasing in $g(\omega)$. Increasing the uncertain parameter leads to increasing in $g(\omega)$ if the following inequality holds:

$$
\left(P_{r}^{2}-P_{i}^{2}+2 \omega P_{r} P_{i}\right) d P_{r}+\left(P_{i}^{2} \omega-P_{r}^{2} \omega+2 P_{r} P_{i}\right) d P_{i}>
$$

$$
0 \quad \text { (11) }
$$

\section{Assumption 1: Let}

$$
P_{i}^{2} \omega-P_{r}^{2} \omega+2 P_{r} P_{i}>0 .
$$

and note that the symbol ' $>$ ' could be inversed; but for simplicity it is assumed as ' $>$ '. Finally, consider the real rational symbol of (12). So (11) can be transformed to

$$
\frac{d P_{i}}{d P_{r}}>\frac{\left(P_{i}^{2}-P_{r}^{2}-2 \omega P_{r} P_{i}\right)}{\left(P_{i}^{2} \omega-P_{r}^{2} \omega+2 P_{r} P_{i}\right)}
$$

and by change of variable as:

$$
z=\frac{P_{i}}{P_{r}}
$$

the inequality (13) can be changed to:

$$
P_{r} d z>\frac{-z^{3} \omega-z^{2}-z \omega-1}{z^{2} \omega-\omega+2 z} d P_{r} .
$$

Assumption 2: Let

$$
P_{r}>0
$$

and

$$
\frac{-z^{3} \omega-z^{2}-z \omega-1}{z^{2} \omega-\omega+2 z}>0
$$

similar to Assumption 1, the real rational symbol of (15) and (16) will be considered finally. From (15) and (16), the inequality (14) leads to:

$$
\ln \left(\frac{-z \omega-1}{\left(z^{2}+1\right) P_{r}}\right)>0 \Rightarrow \frac{-z \omega-1}{\left(z^{2}+1\right) P_{r}}>1
$$

and since $z=\frac{P_{i}}{P_{r}}$, then

$$
p_{i}^{2}+P_{r}^{2}+P_{i} \omega+P_{r}<0
$$

that is one of the necessary conditions imply that increasing the uncertain parameter $q$ leads to increasing in $g(\omega)$. Assumptions 1 and 2 imply another necessary condition as

$$
-\left(P_{r}+P_{i} \omega\right)>0 \text {. }
$$

Remark 2: Bode diagrams corresponding to $P\left(j \omega, q_{\min }\right)$ and $P\left(j \omega, q_{\max }\right)$ could be recognized from the frequency responses set of uncertain plant $P(j \omega, q)$. In fact if (9) or (10) holds, then from monotonic variation of the functiong $(\omega)$, it could be deduced that the uppermost and lowermost plots of $g(\omega)$ are corresponding to $P\left(j \omega, q_{\min }\right)$ and $P\left(j \omega, q_{\max }\right)$, not necessarily respectively.

The following Corollary shows that the problem can be solved easier when the uncertain parameter is in the feedforward path of control loop.

Corollary 1. Let $P(s, q)$ be a real rational function for an uncertain LTI plant and $q \in\left\{q_{\min }, q_{\max }\right\}$ is an uncertain parameter that appears in the feedforward path. Then the family of stabilizing PID controllers for uncertain plant is a subset of common set between two set of stabilizing PID parameters for $P\left(s, q_{\min }\right)$ and $P\left(s, q_{\max }\right)$ if one of the below sets satisfy

$\left\{\begin{array}{l}\left(-P_{r}+\omega P\right)_{i}>0 \text { : if } g(\omega) \text { is ascendant } \\ \left(-P_{r}+\omega P\right)_{i}<0 \text { : if } g(\omega) \text { is decendant }\end{array}\right.$

(17)

for $\omega \geq 0$ and for only one value of uncertain parameter $q$.

proof: It can be written

$P(j \omega, q)=q P_{r}(j \omega)+q P_{i}(j \omega)$

and

$g(\omega)=-\frac{q P_{r}(j \omega)+\omega q P_{i}(j \omega)}{q^{2}\left(P_{r}(j \omega)+P_{i}(j \omega)\right)}$.

Then

$\frac{d g}{d q}=\frac{1}{q^{2}} \cdot \frac{P_{r}+\omega P_{i}}{P_{r}^{2}+P_{i}^{2}}$

So $g(\omega)$ is monotonic if (17) satisfies.

The procedure for calculating the family of stabilizing PID controllers is summarized in the below algorithm.

\section{Algorithm 2.}

1. Determine $P\left(j \omega, q_{\min }\right)$ and $P\left(j \omega, q_{\max }\right)$ for $\omega \geq 0$ from $g(\omega)$ plots using Remark 2.

2. Using Algorithm 1, calculate the family of stabilizing PID controllers for two plants $P\left(s, q_{\min }\right)$ and $P\left(s, q_{\max }\right)$. Determine the common space of PID parameters between two calculated family. The family of stabilizing PID controllers for uncertain plant $P(s, q)$ is a subspace of this common space.

3. Calculate the exact family of stabilizing PID controllers using the approach proposed in Remark 1.

Now it can be shown that the problem of satisfying some performance specifications for uncertain plant $P(s, q)$ could be transformed to problem of robust stabilizing the uncertain plant $P(s, q)$ with additional virtual uncertain parameter.

Many performance attainment problems for uncertain plant $P(s, q)$ can be cast as the simultaneously stabilization of the uncertain plant and the family of real and complex plants [11]. For example an $H_{\infty}$-norm achievment on the complementary sensitivity function, that is $\|W(s) T(s)\|<\gamma$, is equivalent to simultaneously stabilizing the uncertain plant $P^{C}(s, q, \theta)$ as 
$P^{C}(s, q, \theta)=\left\{\left(1+\frac{1}{\gamma} e^{j \theta} W(s)\right) P(s, q): \theta \epsilon\left[\theta_{\text {min }}, \theta_{\text {max }}\right]\right\}$

where $\theta$ is a virtual uncertain parameter and $W(s)$ is a weight selected by designer.

Let the family of stabilizing PID parameters for uncertain plant $P(s, q)$ can be calculated from Algorithm 1 and Let $P^{C}(s, q, \theta)$ in (18) be a real rational function. If $g(\omega)$ corresponding to (18), varies monotonically by monotonic varying the virtual uncertain parameter $\theta$, for any $q$ and any $\theta$, then the family of stabilizing PID controllers for uncertain plant $P(s, q)$ that satisfy the $H_{\infty}$-norm specification on the complementary sensitivity function, is a subspace of PID controllers obtained from simultaneously stabilization of two plants

$$
\begin{array}{r}
P^{C}\left(s, q_{\min }\right)=\left[1+\frac{1}{\gamma} W(s)\right] P \\
P^{C}\left(s, q_{\max }\right)=\left[1+\frac{1}{\gamma} W(s)\right] P\left(s, q_{\max }\right)
\end{array}
$$

and the exact family can be obtained using the approach proposed in Remark 1.

\section{A REVIEW ON THE APPLICATIONS OF THE PROPOSED APPROACH}

In this section, some applications of the proposed method are presented. In fact, it is illustrated that some control objectives can be cast as robust stabilizing of a plant with an uncertain parameter. For example:

\section{Example 1: Performance achievement} Many performance achievement problems for uncertain plant $P(s, q)$ can be cast as the simultaneously stabilization of the uncertain plant and the family of real and complex plants. Some of these performance achievement problems for nominal plant are listed in [11]; for example, the problem of $H_{\infty}$-norm achievement on the complementary sensitivity function is equivalent to simultaneously stabilizing the plant $P(s)$ and the family of real plants

$$
P^{C}(s, q, \theta): q \in\left\{q_{\min }, q_{\max }\right\}, \theta \in\{0,2 \pi\}
$$

and the exact family can be obtained using some test points. The other specifications such as $H_{\infty}$ norm achievement on the sensitivity function and phase margin can be satisfied by the same approach.

\section{Example 2: Robustness against loss of effectiveness in actuator}

Fig. 3 Shows the feedback structure of a plant with PID faced to loss of effectiveness in actuator where $L$ is the parameter corresponding to loss of effectiveness and belongs to $(0,1]$. Obviously this structure can be cast as a plant with an uncertain parameter, i.e., $P(j \omega, L)$ with PID where $L=q$ is the uncertain parameter. There is similar case when loss of effectiveness happens in sensors. Thus the family of robust PID controllers for plants faced to loss of effectiveness can be calculated by the approach proposed in the previous section. For this special case, the problem of controller synthesis could be handled easily using Corollary 1.

\section{Example 3: Robustness in plants with a dominant uncertain parameter}

Some plants that are faced to parameter variations can be approximated by plants with a dominant uncertain parameter. Obviously the proposed approach can be applied in this case to calculate the family of robust stabilizing PID controller.

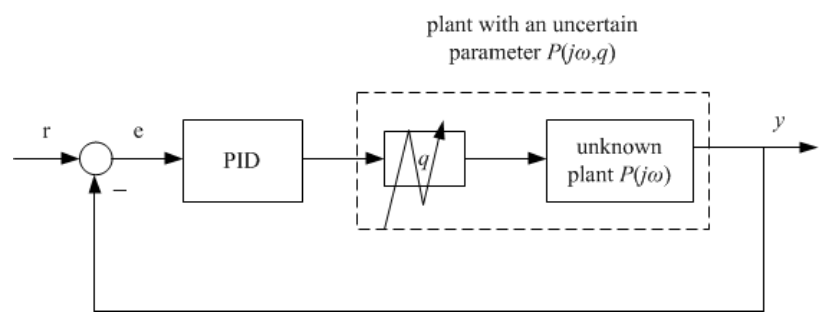

Fig. 3. The feedback structure of plant faced to loss of actuator effectiveness with PID

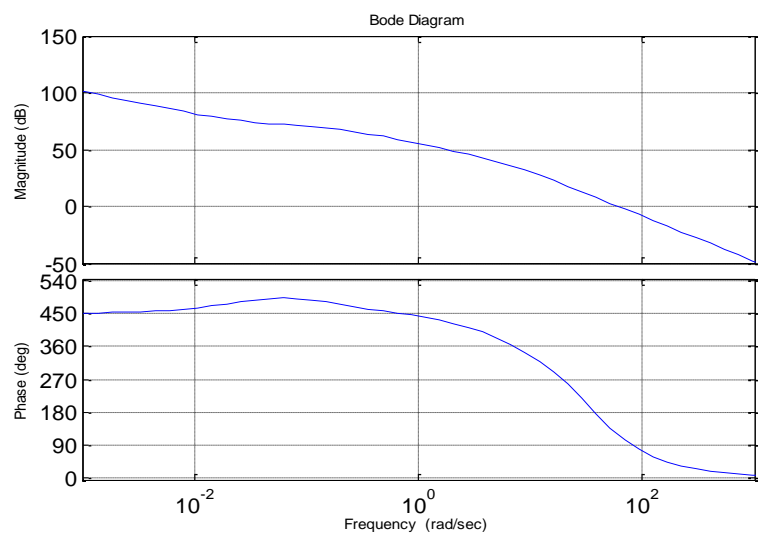

Fig. 4. The frequency response of motor drive system

\section{SIMULATION ON INDUCTION MOTOR}

The model studied in this section is an induction motor drive system introduced in $[9,12]$ that has the similar structure to Fig. 1 in [14] and its nominal frequency response is shown in Fig. 4. The frequency response of such systems can be obtained by virtual sine sweeping [13]. The corresponding plot of $g(\omega)$ is shown in Fig. 5. Since $R \geq 3$ (from (8)), the admissible range of $k_{p}$ is $[-25,395]$. Calculating the $\left(k_{i}, k_{d}\right)$ values for $k_{p}=$ 50 and $T=1$ is resulted to the following inequalities

$$
\left\{\begin{array}{c}
k_{i}>0 \\
k_{i}<16.24 k_{d}+270 \\
k i>370.17 k_{d}-21611 \\
k_{i}<6561 k_{d}+15379
\end{array}\right.
$$



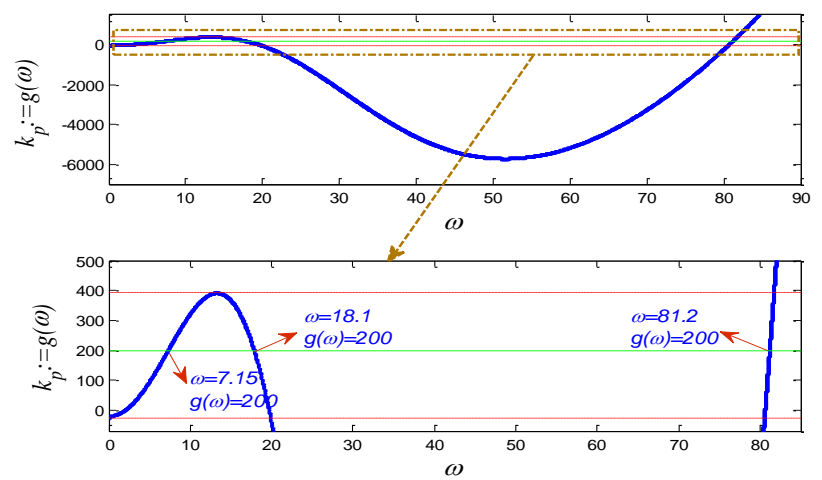

Fig. 5. The plot of function $g(\omega)$ with the line $k p=200$

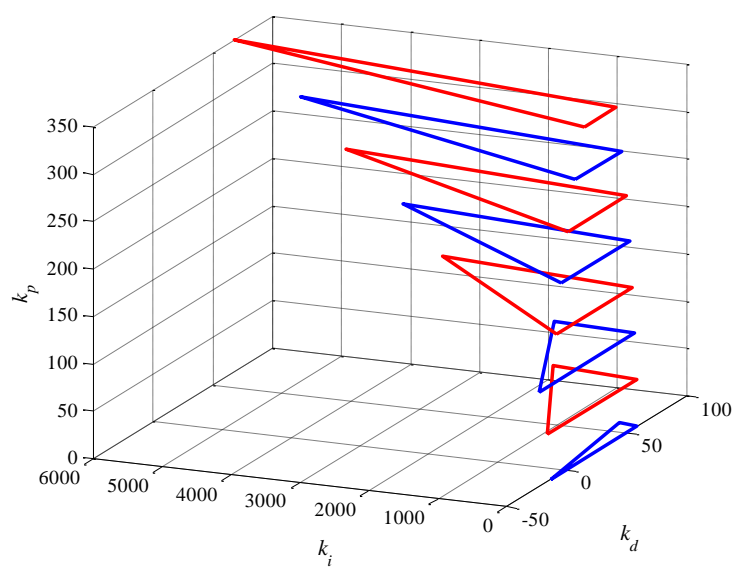

Fig. 6. The whole family of stabilizing PID controllers for nominal model of induction motor drive system

Therefore the family of robust PID controllers for the induction motor drive with uncertainties can be calculated by stabilizing the following two plants

$$
\left\{\begin{array}{l}
\left(1+W(s) \Delta_{\min }\right) P(s) \\
\left(1+W(s) \Delta_{\max }\right) P(s)
\end{array} .\right.
$$

The range of robust stabilizing PID parameters for induction motor faced to un-modeled dynamics is illustrated in Fig. 7-a. The admissible range of $\left(k_{i}, k_{d}\right)$ for all admissible $k_{p}$ is shown in Fig. 7-b.

(a)

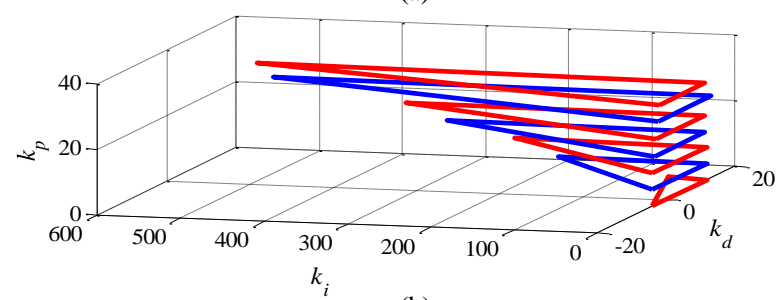

(b)

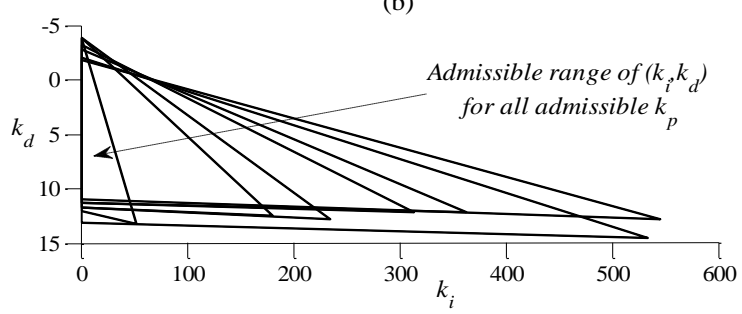

Fig. 7. (a) Robust stabilizing PID parameters for induction motor drive faced to un-modeled dynamics; (b) the admissible range of $\left(k_{i}, k_{d}\right)$

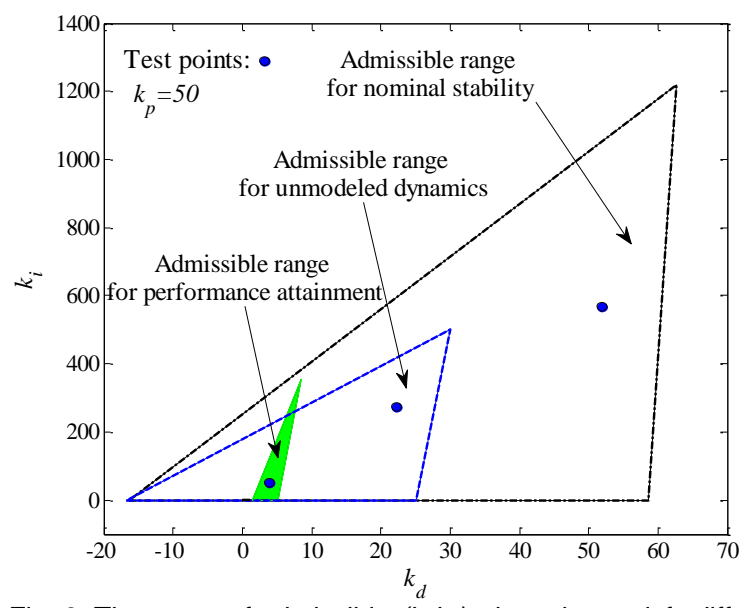

Fig. 8. The range of admissible $\left(k_{i}, k_{d}\right)$ values that satisfy different control objectives for $k_{p}=50$

Now consider the problem of satisfying $H_{\infty}$-norm on the complementary sensitivity function. The admissible range of $\left(k_{i}, k_{d}\right)$ values that satisfy this performance criterion for $k_{p}=50$ is shown in Fig. 8 together with regions corresponding to nominal stability and stability in the presence of un-modeled dynamics. The whole range of $\left(k_{i}, k_{d}\right)$ values can be obtained similarly.

The step responses of closed loop system with controllers corresponding to test points marked in Fig. 8 are shown in Fig. 9. Applying the PID controller that satisfies performance consideration is resulted to decreasing the overshoot. Also, the control signals corresponding to selected controllers are plotted in Fig. 10. It can be seen that the control signals drift to zero after the reasonable times.

For better tracking of the proposed approach in this paper, the readers can refer to the many academic examples implemented in [15].

\section{CONCLUSION}

In this paper, a robust control approach presented based on a general model for different types of electrical motor drives. The problem of robustness against un-modeled dynamics in motor drives is transformed to the problem of stabilizing a plant with an uncertain parameter. It is shown that knowing the frequency responses of motor drive system corresponding to maximum and minimum values of uncertainty is sufficient to calculate the family of robust stabilizing PID controllers. In fact, there is no need to plant mathematical model. Also it is illustrated that the problem of $H_{\infty}$-norm achievement on the complementary sensitivity function can be solved by the same approach. Through the paper, it is assumed that the frequency response of plant is available. Such an assumption is often valid in many practical applications. Also, this is an assumption that has already been used several times in other papers dealing with controller synthesis using frequency domain data $[5,7,11,13,15-17]$. 
There are open doors to extend this approach to MIMO and nonlinear systems and to improve the performance specifications. Also, it is obvious that many of un-modeled dynamics are more complicated than the constant parameter $\Delta$ considered in this paper. So it is of essential interest to extend the approach to more complicated types of uncertainties.

(a)

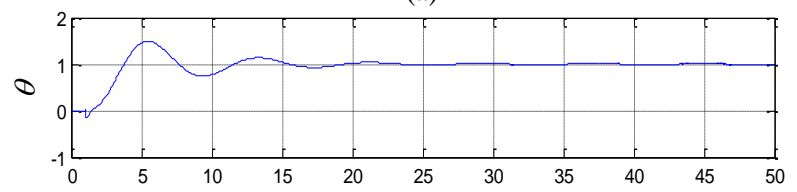

(b)

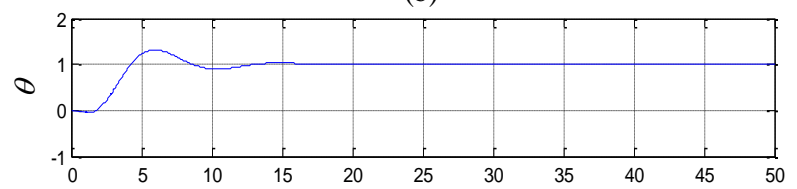

(c)

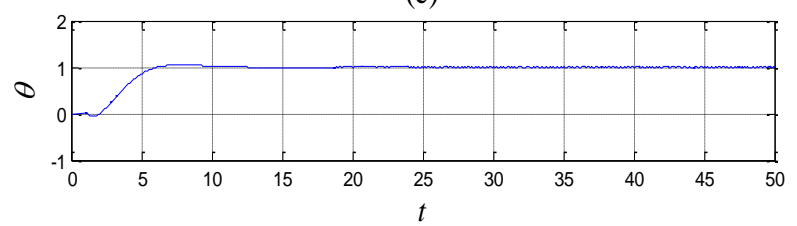

Fig. 9. Step responses corresponding to controllers that satisfy (a) stability for nominal motor drive system, (b) stability for motor drive with un-modeled dynamics, (d) stability and performance attainment for motor drive with un-modeled dynamics (The unit of
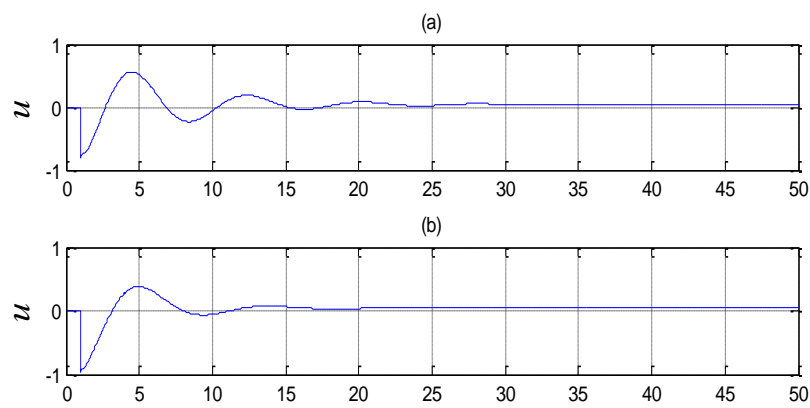

(c)

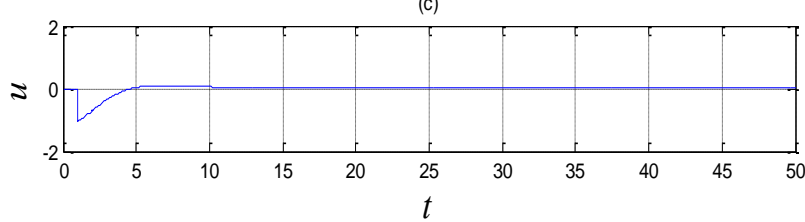

time $t$ is second)

Fig. 10. The control inputs corresponding to controllers that satisfy (a) stability for nominal motor drive system, (b) stability for motor drive with un-modeled dynamics, (d) stability and performance attainment for motor drive with un-modeled dynamics (The unit of time $t$ is second)

\section{REFERENCES}

[1] Shibata, T. and Murakami, T. (2008) Null Space Motion Control by PID Control Considering Passivity in Redundant Manipulator, IEEE trans. Industrial Informatics, vol. 4, no. 4

[2] Ren, T. J., Chen T. C. And Chen,C. J. (2008)Motion Control of a Two-Wheeled Vehicle Using a Self Tunning PID Controller, Contol Engineering Practice, vol. 16, pp. 365-375.
[3] Mikalsen, R., Roskilly, A. P. (2010) The Control of a Free-Position Generator. Part 2: Engine Dynamics and Piston Motion Control, Journal of Applied Energy, pp. 1281-1287.

[4] Ferretti,G., Magnani, G. and Rocco, P. (2001) Alternative in Precise Load Motion Control of Two Mass Servomechanisms, IEEE/ASME International Advanced Parastvand Intelligent Mechatronics Proceedings, Como, Italy.

[5] Parastvand, H. and Khosrowjerdi, M. J. (2015) Controller Synthesis Free of Analytical Model: Fixed Order Controllers, International Journal of Systems Science, vol. 46, no. 7, pp. 1208-1221.

[6] Ouyang, P. R., Pano, V. and Dam, T. (2015) PID Position Domain Control for Contour Tracking, International Journal of Systems Science, vol. 46, no. 1, pp. 111-124.

[7] Parastvand, H. and Khosrowjerdi, M. J. (2014) A New Data-Driven Approach to Robust PID Controller Synthesis, Journal of Control Engineering and Applied Informatics, vol. 16, no. 3, pp. 84-93,.

[8] Parastvand, H. and Khosrowjerdi, M. J.(2015) Parameterized Controller Synthesis for SISO-LTI Uncertain Plants Using Frequency Domain Information, International Journal of Systems Science, in press,.

[9] Lin,F. J., Chen, S. Y., Teng L. T. and Chu, H. (2009) Reurrent Functional Link Based Fuzzy Neural Network with Improved Practical Swarm Optimization for A Linear Synchronous Motor Drive, IEEE trans.Magnetics, vol. 45, no. 8,.

[10] Masiala, M., Vafakhah, B., Salmon J. and Knight A (2008) Fuzzy Self Tunning Speed Control of An Indirect Field Oriented Control Induction Motor Drive, IEEE trans. Industry Applications, vol. 44, no. 6.

[11] Keel, L. H. and Bhattacharyya, S. P. (July 2008) Controller Synthesis Free of Analytical Model: Three term controllers, IEEE trans. Automatic Control, vol. 53, no. 6.

[12] Liu Z. and Wang, Q. (2008) Robust Control of Electrical Machines with Load Uncertainty, Journal of Electrical Engineering, vol. 2, no. 2, pp. 110-112.

[13] Taghirad, H. D. (1997) Robust Torque Control of Harmonic Drive System, Ph.D. dissertation, Dept. Electrical Eng., Univ. McGill, Montreal.

[14] Wildi, T. (2002) Elecctrical Machines, Drives, and Power Systems, Prentice Hall.

[15] Parastvand, H. (2010) Model Free Controller Synthesis: Frequency Domain Approaches, MSc thesis, Sahand University of Technology,

Department of Electrical Engineering, Iran.

[16] S. Formentin, A. Karimi, S.M. and Savaresi, (2012) On Input Design for Direct Data-Driven Controller Tuning, IFAC Symposium on System Identication, Bruxelles, Belgium.

[17] Silva, G. J., Datta, A. and Bhattacharyya,S. P. (2002) New Results on the Synthesis of PID Controllers, IEEE TRANSACTIONS ON AUTOMATIC CONTROL, VOL. 47 , NO. 2, pp. 24125.

[18] Fliess, M. and Join,C., Riachy, S. (2011) Revisiting Some Practical Issues in The Implementation of Model-Free Control, 18th IFAC World Congress, IFAC WC'2011, Milan, Italy.

[19] Fliess, M. and Join, C. (2009) Model-free Control and Intelligent PID Controllers: Towards a Possible Trivialization of Nonlinear Control, 15th IFAC Symposium on System Identi_cation (SYSID 2009), Saint-Malo, France. 